\title{
Twisted growth and organization of cortical microtubules
}

\author{
Takashi Ishida $\cdot$ Siripong Thitamadee $\cdot$ \\ Takashi Hashimoto
}

Published online: 1 February 2007

(C) The Botanical Society of Japan and Springer 2007

\section{Erratum to: J Plant Res}

DOI 10.1007/s10265-006-0039-y

The author would like to add following acknowledgments to the above-mentioned article.

Acknowledgments We thank Dr. Yasushi Yoshioka (Nagoya University) and Dr. Francine Carland (Yale University) for providing trn1 alleles (twist and lopped) described in the text. The work in the authors' laboratory was partly supported by grants from the Ministry of Education, Culture, Sports, Science and Technology of Japan to TH.

The online version of the original article can be found at http://dx.doi.org/10.1007/s10265-006-0039-y.

T. Ishida $\cdot$ S. Thitamadee $\cdot$ T. Hashimoto $(\bowtie)$

Nara Institute of Science and Technology,

Graduate School of Biological Sciences,

8916-5 Takayama, Ikoma, Nara 630-0192, Japan

e-mail: hasimoto@bs.naist.jp 